\title{
Passive compliant quadruped robot using central pattern generators for locomotion control
}

\author{
Simon Rutishauser, Alexander Spröwitz, Ludovic Righetti and Auke Jan Ijspeert \\ Biologically Inspired Robotics Group, School of Computer and Communication Sciences \\ École Polytechnique Fédérale de Lausanne (EPFL), Switzerland \\ simon.rutishauser@epfl.ch, alexander.sproewitz@epfl.ch, ludovic.righetti@a3.epfl.ch, auke.ijspeert@epfl.ch
}

\begin{abstract}
We present a new quadruped robot, "Cheetah", featuring three-segment pantographic legs with passive compliant knee joints. Each leg has two degrees of freedom - knee and hip joint can be actuated using proximal mounted RC servo motors, force transmission to the knee is achieved by means of a bowden cable mechanism. Simple electronics to command the actuators from a desktop computer have been designed in order to test the robot. A Central Pattern Generator (CPG) network has been implemented to generate different gaits. A parameter space search was performed and tested on the robot to optimize forward velocity.
\end{abstract}

\section{INTRODUCTION}

There exist several completely different approaches to artificial legged locomotion. A first example are two-legged passive dynamic walking machines [1], [2]. These are capable of quite natural looking motion on a slight slope without requiring external control or energy other than the work done by gravity. However these machines have several inherent limitations: they require a precisely defined artificial environment and they are limited to one single walking gait - in fact they correspond closely to the inverted-pendulum model for walking.

A different approach, for increasing versatility and especially stability in rough terrain, is to add a number of actuated degrees of freedom (DOF) per leg. Famous four-legged examples in this category are Tekken II and several of the Titan robots [3], [4], [5]. Titan VIII for example has a reptilelike leg configuration with three DOFs per leg and features a pantograph mechanism. Tekken II on the other hand uses a mammal-like leg configuration with four actively actuated DOFs per leg (hip pitch and jaw, knee and ankle pitch joints) and passive compliant elements at the ankle level to prevent the leg from stumbling [3]. A neural system model, consisting of a CPG and reflexes, is used for controlling Tekken. Impressive results are obtained, especially when walking in rough terrain. However this versatility has a cost in terms of complexity, price and weight.

Passive compliant robots such as Scott II [6], [7], Tekken I [8] and Puppy II [9], [10] situate themselves somewhere between passive walkers and complex actively actuated robots. They feature passive knee joints and actively actuated hip joints. This leg configuration is very similar to the springmass model for running and hopping [11] - it is very simple but still achieves interesting results. A stable rotary gallop gait has been shown for Scott II - with impressive velocities of up to $1.2 \mathrm{~m} / \mathrm{s}$, while Puppy II has been shown to perform a hopping gait. However the passively actuated knee joints limit these two robots to gallop and hopping gaits, since most other gaits require active leg retraction during swing phase.

Our new robot design "Cheetah" is inspired by Puppy II [9], [10]. The goal is to design a robot that performs fast and stable quadruped locomotion using multiple gaits thanks to active leg retraction, has a low energy consumption and is as simple as possible.

The robots most important part is its leg design, which has to fulfill several opposing demands: First and foremost it has to be as lightweight as possible, since the final goal is to achieve high stepping frequencies. For the same reason the number of DOFs per leg has to be as low as possible, two DOFs per leg are sufficient for the most common gaits. We placed all actuators proximal, since accelerating the inertia of a motor along with the leg is not a suitable solution. A final important requirement is to include passive compliance to absorb impacts and temporarily store their energy, the final goal being adaptivity, motion stability in rough terrain and possibly lowered energy consumption.

The combination of these requirements has led to our mammal-like three-segmented leg design, featuring a pantograph mechanism that ensures parallelism between two of the three leg segments and helps keeping the number of degrees of freedom low. As an important enhancement over e.g. Puppy II, which has only one actuated DOF per leg, the leg can be actively retracted by actuating the knee joint. The movement transmission from the proximally mounted motors is done by means of a bowden cable. The resulting leg design is very lightweight, cheap and simple.

The leg trajectories for the different gaits are generated by a Central Pattern Generator (CPG) network [12] based on Hopf oscillators. This particular CPG network has several interesting properties which make it advantageous when compared to a purely sine-based controller: (a) switching gaits is done smoothly by simply replacing the matrix that defines the coupling between the CPGs, (b) both stance- and swing-phase duration can be modified independently and (c) the possibility of easily integrating sensory feedback as a 
future option, in order to increase robustness.

\section{Cheetah's COMPLIANT LEG DESIGN}

\section{A. Leg design objectives}

Cheetah features a lightweight three segment mammallike pantograph leg. The knee-joint is passively compliant through a spring working in parallel with the retraction mechanism. Both hip and knee joints are actuated by proximally mounted actuators (Fig. 1(b)), the goal being to minimise inertia and possibly enabling different dynamic gaits.

\section{B. Three-segment leg length ratios}

A first important decision was use three leg segments, since these have been found to be energetically advantageous [13], [14] over legs with both more and fewer segments.

The relative length of the three leg segments is another important choice - it was decided to follow the recommendations given by [14] as closely as possible, using a relative shank length of 0.45 and a ratio between foot and thigh length of 0.4. These ratios also correspond closely to the length ratios observed for cats such as real cheetahs [15].

\section{Pantograph mechanism}

Using three leg segments should not lead to an unnecessary increase in complexity and number of actuators, since one of the main goals of this design is to be as lightweight as possible. It has been shown that, at least for small mammals, two leg segments ${ }^{1}$ are displaced in a nearly parallel fashion over the whole step cycle [16]. In consequence we decided to implement a pantograph mechanism, constantly ensuring parallelism between these two leg segments. Only one DOF that influences the leg length remains and there is no need to take leg configuration stability into account [17].

The distance between the two parallel leg segments of the pantograph mechanism is a compromise: it cannot be very large since this would require a longer lowest leg segment, but it has to be as large as possible to guarantee mechanical strength.

\section{Fore- and hind leg length}

Taking further inspiration from cats, the hind legs are designed 20\% longer than the fore legs [15] (Fig. 1). Whether this entails advantages over a configuration with equal leg lengths and what these advantages are remains yet to be investigated. A slight offset between fore and hind legs in the coronal plane was created such as to avoid leg collisions even for large step angles (Fig. 1). Leg lengths and other key data characterizing "Cheetah" are listed in Tab. I.

\section{E. Compliance and knee joint actuation}

Having evaluated several construction variants, it was decided to implement compliance by adding a compression spring on the diagonal of the pantograph mechanism (Fig. 2). The spring is guided by a plastic tube. During stance phase the spring is compressed by the external forces acting on

\footnotetext{
${ }^{1}$ Forearm and scapula for the forelimb, the configuration for the hindlimb is slightly different though, depending on the species
}

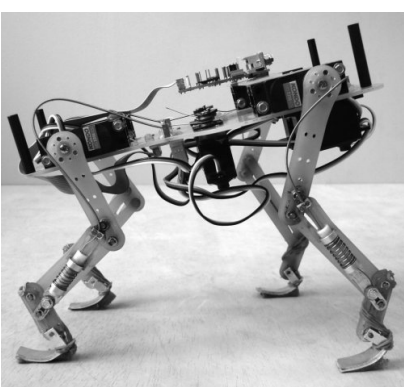

(a) Picture of the real "Cheetah"

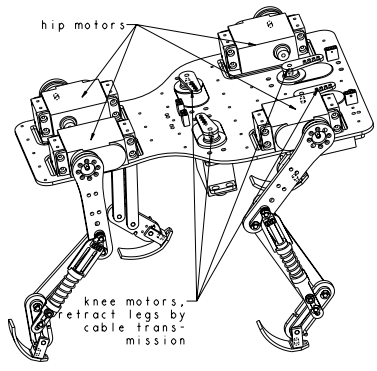

(b) CAD construction drawing
Fig. 1. Picture and CAD drawing of "Cheetah"

TABLE I

CHEETAH'S KEY CHARACTERISTICS

\begin{tabular}{|c|c|}
\hline Property & Value \\
\hline Leg weight (fore/hind leg) & $20 / 23 \mathrm{~g}$ \\
\hline Total weight (with/without battery) & $850 / 720 \mathrm{~g}$ \\
\hline Touchdown leg length (fore/hind leg) & $160 / 190 \mathrm{~mm}$ \\
\hline Robot length & $235 \mathrm{~mm}$ \\
\hline Robot width (fore/hind) & $110 / 170 \mathrm{~mm}$ \\
\hline
\end{tabular}

the leg, thus absorbing parts of the impact energy from touchdown. At the end of the stance phase, before takeoff, it restitutes its energy, thus contributing to the forward motion.

Leg retraction was implemented using a proximally mounted RC servo motor - such as to minimize leg inertia - and a bowden cable for force transmission (Fig. 2). The same Kondo KRS-2350 ICS servo motor is used for hip and knee joint actuation - it provides a stall torque of $2 \mathrm{Nm}$ and a nominal speed of $0.16 \mathrm{~s} / \mathrm{rad}(63 \mathrm{rpm})$.

The foot is an additional compliant element. It can freely rotate around its joint, but is pulled in counterclockwise direction (as in Fig. 2 on the left) using a rubber band. Together with foamed rubber used as foot sole, the elasticity helps to dampen the touchdown impact. In addition it restitutes some of its energy at takeoff. The rubber band as elastic element was chosen over alternative concepts due to its low cost, easy implementation and tuneability.

\section{F. Leg compliance spring dimensioning}

To get a first approximation to the required spring properties, a simple spring-mass model of a single leg was used.

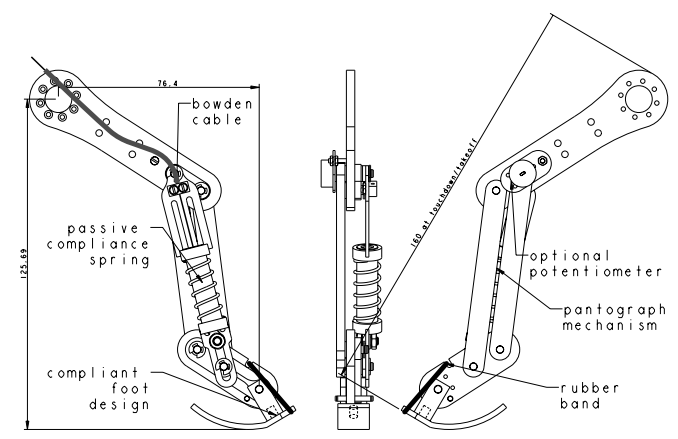

Fig. 2. CAD drawing of the three-segment compliant leg at touchdown or takeoff 
The initial conditions were taken from biological research which suggests stepping frequencies of up to $5 \mathrm{~Hz}$ for mammals of the size of "Cheetah" [18]. The RC servo motors used cannot provide a frequency this high - at most a stepping frequency of about $2.5 \mathrm{~Hz}$ could be achieved; thus this value was used for dimensioning the system. In practice the knee motors proved to be the limiting factor, since they have to move at twice the frequency of the hip motors.

Writing the Lagrange function and using a small-angle approximation, this model can be solved analytically for linear springs [19]. However the pantograph three-segment cannot be represented by a linear spring - due to its geometry it is highly nonlinear (Fig. 3(a)). The large difference between hind and fore leg force is due to the different springs used the fore leg spring constant is $k_{\text {fore }}=0.9 \mathrm{~N} / \mathrm{mm}$ while the hind leg spring constant is $k_{\text {hind }}=2.3 \mathrm{~N} / \mathrm{mm}$. This choice is not only due to the different leg lengths but also due to the somewhat uneven weight distribution on the robot - the hind legs have to bear more of the motors weight.

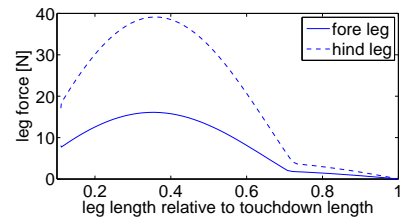

(a) Nonlinear force characteristics of the compliant pantograph leg

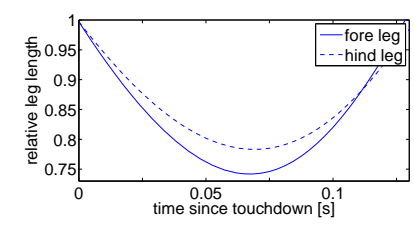

(b) Relative leg length over a stance cycle
Fig. 3. Static leg pantograph elasticity characteristics and dynamic behavior

The numerical solutions of the equations of motion using the nonlinear leg-equivalent spring ${ }^{2}$ (Fig. 3(b)) show that the stance cycle duration for hind- and fore legs is about the same despite the different spring constants, but the fore leg is compressed more.

\section{G. Materials}

The whole leg has to be built as lightweight as possible. All the large structural elements are made of FR4, consisting of glass fibers and epoxy resin. It is quite lightweight, elastic and has appropriate mechanical properties and is readily available for rapid prototyping. Wiring to eventual sensors can be directly integrated on the leg structure, an option that the mechanically even more interesting carbon fiber materials lack.

The only severe limitation of FR4 as building material is that it is only available as thin boards. Two FR4 layers were glued together to increase thickness at the joints of the pantograph mechanism.

\section{H. Electronics}

The electronic setup consists of a PIC-18F2850 board generating the PWM signals for the eight servo motors.

\footnotetext{
${ }^{2}$ The total robot weight is below $1 \mathrm{~kg}$, assuming a trot or pace gait with duty cycle of 0.5 , each leg has to bear the load of about half the robot; the forward velocity is assumed as $v=0.5 \mathrm{~m} / \mathrm{s}$. For a walk gait with longer stance phase the equivalent load is lower, whereas it is higher for a bound gait.
}

Currently the system is run in open-loop mode without any feedback. Control signal generation is done on a desktop PC which sends the setpoints over a serial line.

\section{Central Pattern Generators for locomotion CONTROL}

The control of locomotion is done using a Central Pattern Generator (CPG), inspired from biological CPGs found in animals. They are neural networks responsible for the generation of the complex coordinated control signals during rhythmic movements. For locomotion control, these CPGs are located in the spinal cord in vertebrate animals. Simple control signals from the brainstem can activate them and modulate their behavior (i.e. speed of locomotion, gaits).

In robotics, CPGs (often modeled as networks of coupled oscillators) have been successfully used to generate trajectories for locomotion control. Such systems have interesting stability properties (limit cycle behaviour), perturbations are rapidly smoothed out. Their synchronization capabilities allow the coordination of the robot's different DOFs in order to create different gaits and permit strong coupling to the robot's environment. Additionally they reduce the dimensionality of the control problem in state space: simple parameter changes allow to smoothly modulate locomotion (see [20] for a review).

\section{A. CPG model}

The CPG model we used to control the robot was initially developed in [12]. Although it was successfully tested with several simulated quadruped robots, this is the first time that it is used to control a real robot.

This model is made of coupled oscillators. It is able to generate several different gaits by simple parameter changes, it is also possible to independently control the amplitude of the trajectories and the duration of the ascending and descending phases of the oscillation which is very useful to control the duration of the swing and stance phases. Indeed, it is well-known from quadruped mammal locomotion that the duration of the swing phase is kept constant over ranges of speed and gaits and that the duration of the stance phase controls the speed of locomotion [21]. In [12], numerical simulations showed that the same held for quadruped robots and that the swing duration was important for stability issues.

Furthermore, this CPG model allows integration of sensory feedback for online trajectory generation, to have more robust locomotion (see [12]). Although we do not currently use sensory information, it is important that the controller can easily be extended to integrate such information, since the next goal would be to add sensors to the robot.

The CPG model is made of four coupled oscillators, the equation for an oscillator $i$ is

$$
\begin{aligned}
\dot{x_{i}} & =\alpha\left(\mu-r_{i}^{2}\right) x_{i}-\omega_{i} y_{i} \\
\dot{y_{i}} & =\beta\left(\mu-r_{i}^{2}\right) y_{i}+\omega_{i} x_{i}+\sum k_{i j} y_{j} \\
\omega_{i} & =\frac{\omega_{\text {stance }}}{\mathrm{e}^{-b y}+1}+\frac{\omega_{\text {swing }}}{\mathrm{e}^{\text {by }}+1}
\end{aligned}
$$



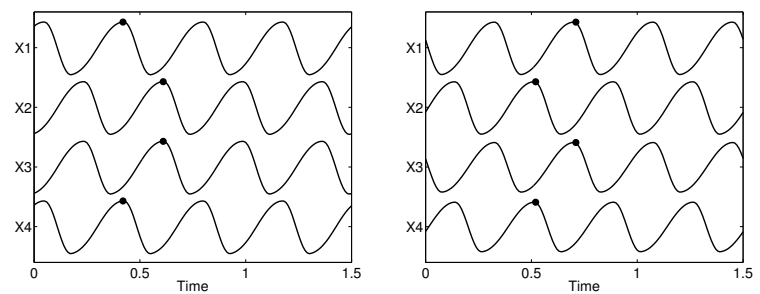

$\left[\begin{array}{cccc}0 & -1 & -1 & 1 \\ -1 & 0 & 1 & -1 \\ -1 & 1 & 0 & -1 \\ 1 & -1 & -1 & 0\end{array}\right]$

Trot
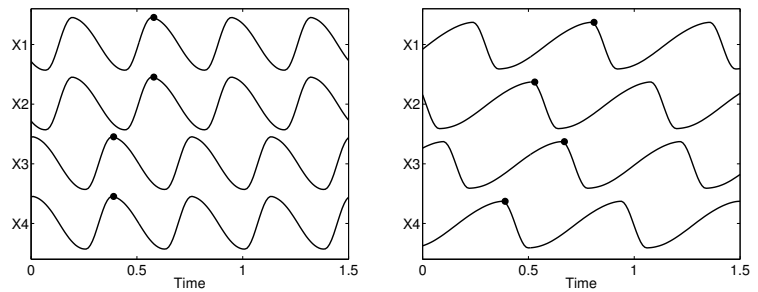

$\left[\begin{array}{cccc}0 & 1 & -1 & -1 \\ 1 & 0 & -1 & -1 \\ -1 & -1 & 0 & 1 \\ -1 & -1 & 1 & 0\end{array}\right]$

Bound

Fig. 4. Coupling matrices and examples of gait generation for the 4 gaits. $\omega_{\text {stance }}=2 \omega_{\text {swing }}$ for the trot and pace gaits. $\omega_{\text {stance }}=4 \omega_{\text {swing }}$ for the walking gait and $\omega_{\text {swing }}=2 \omega_{\text {stance }}$ for the bound.

The variables X1 through X4 correspond to the front left, front right, hind left and hind right leg respectively (reproduced from [12]).

where $r_{i}=\sqrt{x_{i}^{2}+y_{i}^{2}}, \alpha$ and $\beta$ are positive constants defining the time of convergence to the limit cycle, $b$ is a positive constant that is chosen to be high $(b=100$ in our case) and the $k_{i j}$ are coupling constants from oscillators $j$ to $i$. Each oscillator has a structurally stable limit cycle, the variables $x_{i}$ and $y_{i}$ oscillate with amplitude $\sqrt{\mu}$. The frequency of oscillation is defined by $\omega_{i}$, it switches between two durations $\omega_{\text {stance }}$ and $\omega_{\text {swing }}$ that respectively define the durations of the ascending and descending phases of the oscillation (i.e. stance and swing durations).

Figure 4 shows the coupling matrices used to define the coupling structure between the oscillators and examples of the generated patterns. Note that the coupling structure is independent of the internal dynamics of each cell and that any other type of oscillator could be used to generate oscillations with different shapes but with the same phase relationship between the oscillators (i.e. same gait). Smooth transition between gaits is possible by simply changing the coupling matrix as shown in Figure 5(a).

\section{B. Control of the cheetah robot}

The $x$ variable of each oscillator is used directly as angular position setpoint for a hip joint of the robot: $h(x)=x+\delta$, where $\delta$ is an angular offset with respect to the vertical.

The trajectories corresponding to the knee joints are:

$$
k(x, y)= \begin{cases}0 & \text { if } y \geq 0 \\ 10(1-|x|) & \text { if } y<0,|x|>0.9 \\ 1 & \text { else }\end{cases}
$$

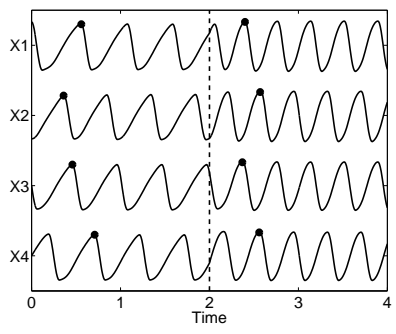

(a)
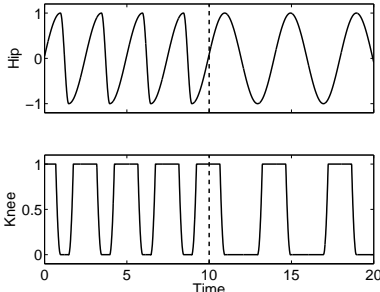

(b)
Fig. 5. Smooth transitions of the trajectories generated by the CPG network: (a) between a walk and a pace gait at $t=2 \mathrm{~s}$ and (b) increase of $\omega_{\text {swing }}$ for the hip and knee joints with at $t=10 \mathrm{~s}$

The function $k(x, y)$ corresponds to the length of the diagonal in the pantograph mechanism (Fig. 2) along the compression spring. It is zero if the leg is completely extended as during stance phase and one if the leg is completely compressed. The relation between $k(x, y)$ and the leg length is nonlinear and depends on the leg geometry, but it is of little interest at the moment since the leg is either fully compressed or extended most of the time.

Figure 5(b) shows typical trajectories sent to the hip and knee joints. In reality the leg trajectories are not as abrupt as the generated setpoints suggest, since they are followed by PID controllers in the RC servo motors that

\section{EXPERIMENTS}

Out of the four possible gaits deriving from the CPG described before, we tested two (walk and pace) on our robot. The CPG is characterized by six parameters: 1) the gait (walk, pace, trot or bound) 2) $\left.\omega_{\text {stance }} 3\right) \omega_{\text {swing }} 4$ ) the amplitude for the oscillation 5) the oscillation offset for the front legs and 6) the oscillation offset for the hind legs.

\section{A. Experimental setup}

For both gaits we did two systematic searches by choosing those two out of the remaining five parameters that had empirically been determined as being the most influential ones (walk: a) $\omega_{\text {stance }}$ and $\omega_{\text {swing }}$, b) $\omega_{\text {stance }}$ and the amplitude. pace: a) $\omega_{\text {stance }}$ and $\omega_{\text {swing }}$, b) $\omega_{\text {stance }}$ and the hind limbs offset). The parameter combinations were tested by running the robot on a $3 \mathrm{~m}$ track, measuring the time in seconds. In case the robot needed more than 1 min for the crossing the track, half the track length was used. Some of the parameter combinations produced a gait destabilizing the robot so that it tumbled over. In these cases, which are marked by cross-outs in the plots, the supply cable was used to stabilize the robot for the tests. In case the robot locomoted in a non-straight pattern, it was moved by laterally pushing it back on the track. A restriction by walls or strings would also be possible, however in such a setup completely instable gait patterns are harder to detect. The parameter range and selection was found by testing neighbouring parameter areas (Tab. II). It presents only a small range of the possible working space and is meant as a preliminary description. Setpoint values for controlling the amplitude and offsets 
TABLE II

PARAMETERS FOR THE SYSTEMATIC SEARCH EXPERIMENTS

\begin{tabular}{|c|c|c|c|c|}
\hline experiment & 1 (Fig. 6 a) & 2 (Fig. 6b) & 3 (Fig. 7 a) & 4 (Fig. 7 b) \\
\hline gait & walk & walk & pace & pace \\
$\omega_{\text {stance }}$ & $3-8 \mathrm{rad} / \mathrm{s}$ & $3-8 \mathrm{rad} / \mathrm{s}$ & $10-15 \mathrm{rad} / \mathrm{s}$ & $7-17 \mathrm{rad} / \mathrm{s}$ \\
$\omega_{\text {swing }}$ & $3-8 \mathrm{rad} / \mathrm{s}$ & 8 & $10-15 \mathrm{rad} / \mathrm{s}$ & 13 \\
amplitude & 60 & $40-90$ & 30 & 30 \\
offset front & 20 & 20 & 30 & 20 \\
offset hind & -10 & -10 & 10 & $5-20$ \\
\hline
\end{tabular}

represent a part of 250 setpoints for a servo motor moving range of $\pi$.

\section{B. Walking gait}

The walking gait (Fig. 8 and [22]) produced the fastest locomotion. With the first search a stable pattern could be found for large $w_{\text {swing }}$, which corresponds a relatively long stance phase (Fig. 6 a). Fixing $\omega_{\text {swing }}$ and alternating the amplitude in the second experiment increased the speed significantly up to almost 1 body length per second. The velocity increases with the step amplitude and $w_{\text {stance }}$ until the motors saturate (Fig. $6 \mathrm{~b}$ ). The unstable areas with higher speed than the stable parameter ranges in Fig. 6 a show that a passive or active stabilization of the gait (possibly a tail or an active back for the robot) could increase the velocity.

\section{Pace gait}

Possibly because of the fixed back the quadruped robot performed less fast in the pace gait (Fig. 9 and [22]) than in the walking gait - about 0.47 body lengths per second. It was also quite hard to find a good range for the systematic search. Finally small amplitudes (half as big as for the walking gait) and relatively high frequencies resulted in stable locomotion (Fig. 7). In case the robot was unstable it tipped over the side, because the legs on each side are working in parallel. Especially for the pace gait, a tail for improving equilibrium of the system might be helpful.

\section{CONCLUSiOn AND Future WORKS}

\section{A. Conclusion}

In this paper we presented a new quadruped robot, "Cheetah", featuring lightweight three-segment pantographic legs. It is inspired by Puppy II [9], [10], cheap and simple when compared to other robots [3], [4], [5], but nevertheless provides two actively actuated DOFs per leg - the hip joint and a passively compliant knee joint, that can be actively actuated through a proximally mounted actuator. Locomotion control has been implemented using a CPG network capable of generating the most common quadruped gaits. The robot has been shown to perform several gaits in experiments ${ }^{3}$, where stability was tested and forward velocity has been optimized in selected regions of the parameters space.

\footnotetext{
${ }^{3}$ walk and pace, trot is also possible and stable but its performance has not yet been systematically investigated
}

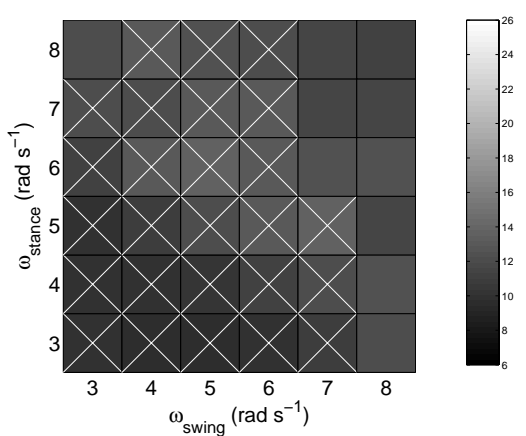

(a)

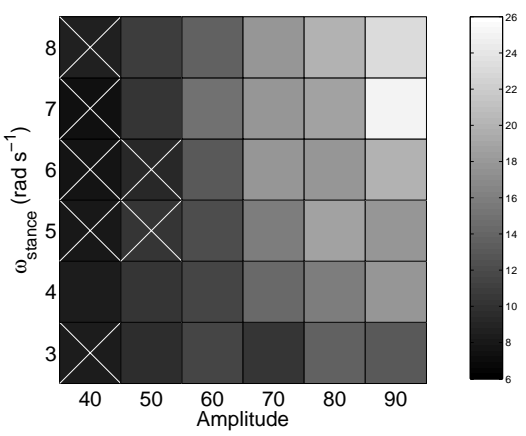

(b)

Fig. 6. Systematic search for the walking gait, two parameters respectively. Open parameters (a): $\omega_{\text {swing }}$ and $\omega_{\text {stance, }}(\mathrm{b}): \omega_{\text {swing }}$ and the shared amplitude for hind and front legs. The speed values are in $[\mathrm{cm} / \mathrm{s}]$ (right column). Crossed out areas correspond to an unstable parameter combination. The maximum speed found for a stable gait is about $25 \mathrm{~cm} / \mathrm{s}$ (about 1 body length per second).

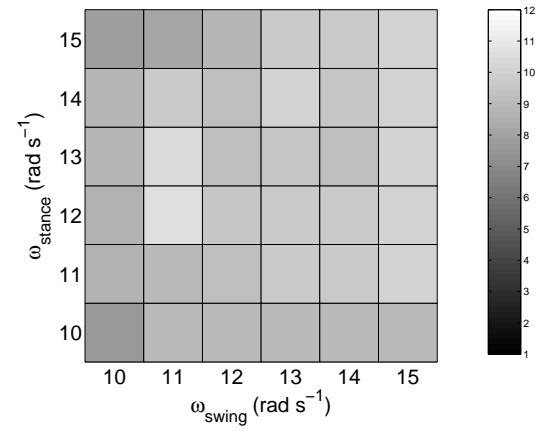

(a)

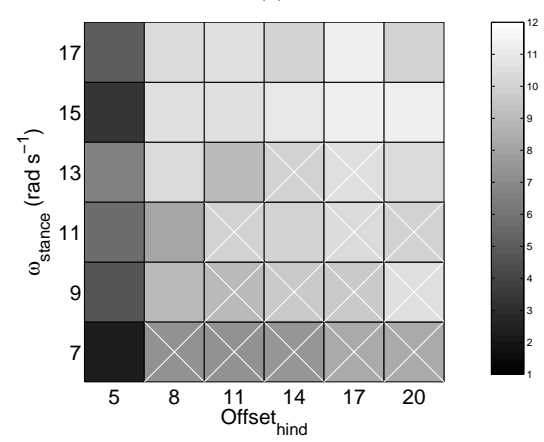

(b)

Fig. 7. Systematic search for the pace gait, two parameters respectively. Open parameters (a): $\omega_{\text {stance }}$ and $\omega_{\text {swing }}$, (b): $\omega_{\text {stance }}$ and hind leg offset. Crossed-out areas correspond to an unstable parameter configuration, the speed values are in $[\mathrm{cm} / \mathrm{s}]$. The maximum speed found for the pace gait is about $11 \mathrm{~cm} / \mathrm{s}$ (about 0.47 body lengths per second). 


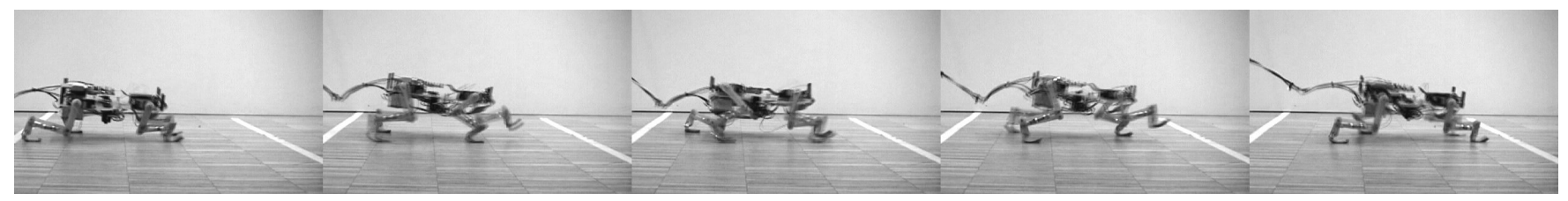

Fig. 8. Snapshots of a walking gait (Movie on [22]). The robot is going from the left to the right. The distance between the two parallel white lines is about $60 \mathrm{~cm}$, about $100 \mathrm{~ms}$ are between each picture. The rather low posture is due to the large leg offsets required to achieve fast and stable locomotion and the weak fore leg springs introduced for the same reason.

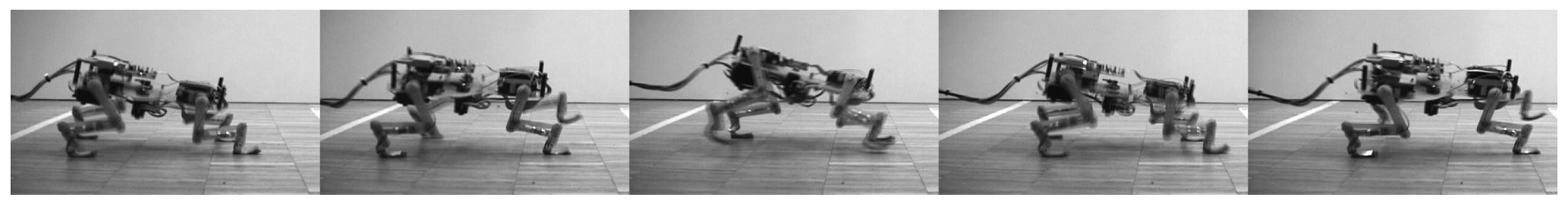

Fig. 9. Snapshots of the robot in pace gait (Movie on [22]). The robot is going from the left to the right. Because the legs on one side of the robot work in parallel (pace gait) the robot is propelled by a rolling motion, by lifting up on side after the other. About 175 ms are between two pictures, the overall sequence takes roughly $0.9 \mathrm{~s}$.

\section{B. Future Works}

There are several directions for future works based on Cheetah: Improving the few weak points in the mechanical design that were discovered when experimenting, making the robot cordless, introducing sensory feedback to the CPGs and adding a flexible but actuated back in order to improve gait stability and increase the robot velocity. Optimization - possibly in a simulation - of the main design parameters such as fore and hind leg length relation, relative leg segment lengths or gear transmission ratio of the motors with regard to the resulting performance are also research topics of interest.

\section{ACKNOWLEDGMENTS}

This work was made possible thanks to financial support from EPFL and the Swiss National Science Foundation. We gratefully acknowledge the technical support of André Guignard, André Badertscher, Philippe Vosseler and Manuel Leitos.

\section{REFERENCES}

[1] T. McGeer, "Passive dynamic walking," International Journal of Robotics Research, vol. 9, no. 2, pp. 62-82, 1990.

[2] A. D. Kuo, "Stabilization of lateral motion in passive dynamic walking," The International Journal of Robotics Research, vol. 18, pp. 917-930, 1999.

[3] H. Kimura and Y. Fukuoka, "Biologically inspired adaptive dynamic walking in outdoor environment using a self-contained quadruped robot: 'tekken2',' Intelligent Robots and Systems, 2004. (IROS 2004). Proceedings. 2004 IEEE/RSJ International Conference on, vol. 1, pp. 986-991 vol.1, 28 Sept.-2 Oct. 2004.

[4] K. Arikawa and S. Hirose, "Development of quadruped walking robot titan-viii," Intelligent Robots and Systems '96, IROS 96, Proceedings of the 1996 IEEE/RSJ International Conference on, vol. 1, pp. 208214 vol.1, 4-8 Nov 1996.

[5] S. Hirose and K. Arikawa, "Coupled and decoupled actuation of robotic mechanisms," Advanced Robotics, vol. 15, no. 2, pp. 125-138, 2001

[6] D. Papadopoulos and M. Buehler, "Stable running in a quadruped robot with compliant legs," Robotics and Automation, 2000. Proceedings. ICRA 'OO. IEEE International Conference on, vol. 1, pp. 444-449 vol.1, 2000 .
[7] J. Smith and I. Poulakakis, "Rotary gallop in the untethered quadrupedal robot scout ii," Intelligent Robots and Systems, 2004. (IROS 2004). Proceedings. 2004 IEEE/RSJ International Conference on, vol. 3, pp. 2556-2561 vol.3, 28 Sept.-2 Oct. 2004.

[8] Z. Zhang, Y. Fukuoka, and H. Kimura, "Stable quadrupedal running based spring-loaded two-segment legged on a model," Robotics and Automation, 2004. Proceedings. ICRA '04. 2004 IEEE International Conference on, vol. 3, pp. 2601-2606 Vol.3, 26 April-1 May 2004.

[9] F. Iida, "Cheap design approach to adaptive behavior: Walking and sensing through body dynamics," in International Symposium on Adaptive Motion of Animals and Machines, 2005.

[10] F. Iida, G. Gomez, and R. Pfeifer, "Exploiting body dynamics for controlling a running quadruped robot." in Proceedings of the 12th Int. Conf. on Advanced Robotics (ICAR05), 2005, pp. 229-235.

[11] R. Blickhan, "The spring-mass model for running and hopping," Journal of Biomechanics, vol. 22, no. 11-12, pp. 1217-1227, 1989.

[12] L. Righetti and A. Ijspeert, "Pattern generators with sensory feedback for the control of quadruped locomotion," in Proceedings of the 2008 IEEE International Conference on Robotics and Automation (ICRA 2008), 2008, conference, in Press.

[13] M. Günther, V. Keppler, A. Seyfarth, and R. Blickhan, "Human leg design: optimal axial alignment under constraints," Journal of Mathematical Biology, vol. 48, no. 6, pp. 623-646, 2004.

[14] R. Blickhan, A. Seyfarth, H. Geyer, S. Grimmer, H. Wagner, and M. Günther, "Intelligence by mechanics," Philosophical Transactions of the Royal Society, vol. 365, pp. 199-220, 2007.

[15] L. M. Day and B. C. Jayne, "Interspecific scaling of the morphology and posture of the limbs during the locomotion of cats (Felidae)," $J$ Exp Biol, vol. 210, no. 4, pp. 642-654, 2007.

[16] M. S. Fischer, N. Schilling, M. Schmidt, D. Haarhaus, and H. Witte, "Basic limb kinematics of small therian mammals," J Exp Biol, vol. 205, no. 9, pp. 1315-1338, 2002.

[17] A. Seyfarth, M. Günther, and R. Blickhan, "Stable operation of an elastic three-segment leg," Biological Cybernetics, vol. 84, no. 5, pp. 365-382, 2001.

[18] R. M. Alexander, "The Gaits of Bipedal and Quadrupedal Animals," The International Journal of Robotics Research, vol. 3, no. 2, pp. 49-59, 1984

[19] H. Geyer, A. Seyfarth, and R. Blickhan, "Spring-mass running: simple approximate solution and application to gait stability," J. Theor. Biol., vol. 232, no. 3, pp. 315-328, 2004.

[20] A. J. Ijspeert, "Central pattern generators for locomotion control in animals and robots: a review," Neural Networks, vol. 21, no. 4, pp. 642-653, 2008.

[21] S. Grillner, "Locomotion in vertebrates: central mechanisms and reflex interaction," Physiol. Rev., vol. 55, no. 2, pp. 247-304, 1975.

[22] (2008) Cheetah movies. [Online]. Available: http://birg2.epfl.ch/movies/full/avi/cheetah/Cheetah_walk_xvid.avi and http://birg2.epfl.ch/movies/full/avi/cheetah/Cheetah_pace_xvid.avi 